TITLE:

\title{
Regularization of the Product of Complex Powers of Polynomials and its Application
}

$\operatorname{AUTHOR}(\mathrm{S})$ :

SEKIGUCHI, Jiro

CITATION:

SEKIGUCHI, Jiro. Regularization of the Product of Complex Powers of Polynomials and its Application. 数理解析研究所講究録 1988, 642: 157-179

\section{ISSUE DATE:}

1988-02

URL:

http://hdl.handle.net/2433/100210

RIGHT: 


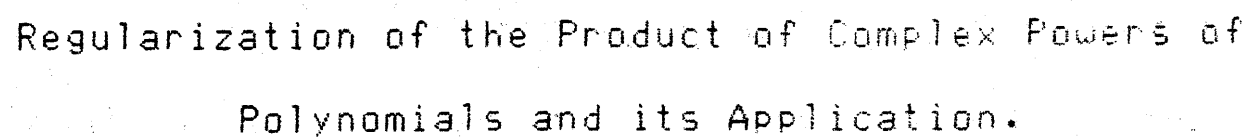

Polynomials and its Application.

\author{
Jiro SEKIGUCHI \\ Department of Mathematics \\ University of Electra-Communications \\ Chofu 182, Tokyo, JAPAN
}

(電通大関口次郎)

Dedicated to Professor M. Sato for his sixtieth birthday

Introduction.

The meromorphic property of complex powers of polynomials established by Bernstein-Gel' fand $[B G]$ is a powerful tool in the construction of hyperfunctions or distributions invariant under group actions. For example, one may recall the construction of fundamental solutions of partial differential equations with constant coefficients, those of relative invariant hyperfunctions on prehomogeneous vector spaces and so on. The original proof of the result of Bernstein-Gel'fand is based on Hironaka's desingularization theorem. Later, a simpler proof was given by I. N. Bernstein [B], showing the existence of b-functions (= Bernstein-Sato polynomials) of polynomials on $\mathbb{C}^{n}$. It is stressed here that it is $M$. Sato who showed the existence of b-functions of relative invariant polynomials of prehomogeneous vector spaces and conjectured the existence of b-functions of arbitrary polynomials in early 1960's. An extension to the case of holomorphic functions were shown by 
$J-E$. Björk $[B j]$ and M. Kashiwara $[K]$.

Let $G / H$ be a semisimple symmetric space. Then to construct Poisson kernels on $G / H$ is equivalent to do hyperfunctions on $G$ which are invariant under left $H$-action and "relative invariant" under right action of an appropriate parabolic subgroup of $G$. The latter is reduced to the problem of regularizing of distributions which are defined by the product of complex powers of analytic functions on $G$. So it is possible to apply the result of Bernstein-Gel'fand mentioned above. Such distributions play an important role in the study of representations of $G$ because they generate principal series representations for $G / H$ (cf. [02]).

We now explain the contents shortly. In $\$ 1$, we shall formulate a generalization ( $c f$. Theorem 1) of the result of $I$. N. Bernstein and S. I. Gel' fand [BG] to the case of $\mathbb{C}$-valued polynomials under an assumption (Theorem 1, condition ( $A$ )) concerning the continuity of complex powers of $\mathbb{C}$-valued polynomials. This assumption seems less trivial to check for given polynomials. Section 2 is devoted to an application of Theorem 1. Let $\widetilde{G}$ be the universal covering group of SU $(p+q, p)$ and let $\tilde{H}$ be a connected closed subgroup of $\tilde{G}$ whose Lie algebra is $s o(p+q, q)$. Introduce real analytic functions $\tilde{f}_{j}(g)(1 \leq j \leq 2 p+q-1)$ on $\widetilde{G}$ which are left $\hat{H}$-invariant. As an application of Theorem 1, it will be shown in Theorem 9 that if $\ell_{1}, \ldots, \ell_{p-1} \in \mathbb{Z}, m_{1}, \ldots, m_{q} \in \mathbb{N}$, then

$$
\zeta_{\lambda, \alpha, e^{(g)}}=\bar{f}_{p}(g)^{\alpha \bar{f}_{p}(g)^{8}} \prod_{j=1}^{p-1}\left|f_{j}(g)\right|^{\lambda} \gamma_{f}(g)^{\ell} j \prod_{k=1}^{q} f_{p+k}(g)^{m_{k}}
$$


defines distributions on $\tilde{G}$ depending on complex parameters $\alpha$, $\beta, \lambda_{1}, \ldots, \lambda_{p-1}$ meramorphically. The key to its proof is to check the conditon (A) of Theorem 1. In this paper, we do not explain the role of $\zeta_{\lambda, \alpha, \beta^{(g)}}$ in the study af analysis on semisimple symmetric spaces. The reader who is interested in this subject, consult $[0 \$ 1,2]$. In $\$ 3$, we restrict aur attention to the case $P=1$, that is, $\widetilde{G}=\operatorname{SU}(q+1,1)^{\sim}$ and study spherical functions $\phi_{\alpha, \beta}(g)$ on $\widetilde{H} \backslash \widetilde{G}$ which are abtained

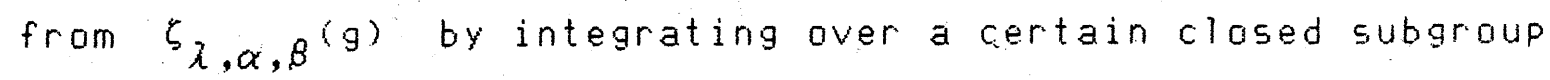
of $\widetilde{G}$ (cf. $\$ 3,(1))$. In particular, we shall prove Theorem 12 which states that $\phi_{\alpha, \beta}$ is expressed in terms of Gaussian hypergeametric functions. In Remark 14, a connection between the study of $\phi_{\alpha, \beta}(g)$ and c-functions for semisimple symmetric spaces is explained.

$\$ 1$. Meromorphic continuation of distributuions defined by the product of complex powers of polynomials

Let $P\left(x_{1}, \ldots, x_{n}\right)$ be an $R$-valued polynomial. Then the function $|P(x)|^{\lambda}$ is continuous if $\operatorname{Re} \lambda>0$, and as a function of $\lambda$, it is extended to a $\sigma^{-}\left(\mathbb{R}^{n}\right)$-valued meromorphic function on c. This is a result of I. N. Bernstein-S. I. Gel' fand [BG]. An extension of the meromorphic property of $|P(x)|^{\lambda}$ to the case of $\mathbb{C}$-valued polynomials is formulated in the following manner.

Theorem 1. Let $f_{1}(x), \ldots, f_{\ell}(x)$ be $\mathbb{R}$-valued polynomials 
and let $g_{1}(x), \ldots, g_{m}(x)$ be $\mathbb{C}$-valued polynomials of $x=\left(x_{1}, \ldots, x_{n}\right)$. Let $\Omega$ be a connected component of the set $\left\{x \in \mathbb{R}^{n} ; f_{i}(x) \neq 0(1 \leq i \leq l), g_{j}(x) \neq 0(1 \leq j \leq m)\right\}$ and take $p \in \Omega$ and $f i x i t$. Suppose that $f_{i}(p)=1(1 \leq i \leq \ell)$, $g_{j}(p)=1 \quad(1 \leq j \leq m)$.

Assume the condition:

(A) Take $\lambda_{i} \in \mathbb{C}(1 \leq i \leq \ell), \mu_{j}, \nu_{j} \in \mathbb{C}(1 \leq j \leq m)$ such that $\operatorname{Re} \lambda_{i}>0, \operatorname{Re}\left(\mu_{j}+\nu_{j}\right)>0$. Then each branch of the function $\prod_{i=1}^{\ell}\left|f_{i}(x)\right|^{\lambda} i \prod_{j=1}^{m} g_{j}(x)^{\mu} j{\frac{g_{j}(x)}{\nu} j}_{j}$ on $\Omega$ is single-valued.

Let $\phi_{\lambda, \mu, \nu}(x)$ be the branch of the function $\prod_{i=1}^{\ell}\left|f_{i}(x)\right|^{\lambda} \prod_{j=1}^{m} g_{j}(x)^{\mu}{\frac{1}{g_{j}(x)}}^{\nu} j$ such that $\phi_{\lambda, \mu, \nu}(p)=1$. Define a function $\phi_{\lambda, \mu, \nu}(x)$ on $\mathbb{R}^{n}$ so that $\phi_{\lambda, \mu, \nu}(x)=\phi_{\lambda, \mu, \nu}^{-}(x)$ if $x \in \Omega$ and $\phi_{\lambda, \mu, \nu}(x)=0$ if otherwise. Then the following hold.

(i) For a non-negative integer $P$, define $S(P)=\left\{(\lambda, \mu, \nu) \in \mathbb{C}^{\ell+2 m} ; \operatorname{Re} \lambda_{i}>P(1 \leq i \leq \ell) \cdot \operatorname{Re} \mu_{j}>p\right.$, $\left.\operatorname{Re} \nu_{j}>p(1 \leq j \leq m)\right\}$. Then $\phi_{\lambda, \mu, \nu}(x)$ is of class $C^{P}$ if $(\lambda, \mu, \nu) \in S(p)$. Moreover, as a function of $(\lambda, \mu, \nu), \phi_{\lambda, \mu, \nu}(x)$ is holomarphic on the open subset $5(0)$ of $\mathbb{C}^{\ell+2 m}$.

(ii) $\phi_{\lambda, \mu, \nu}(x)$ is extended to a $\sigma^{-}\left(\mathbb{R}^{n}\right)$-valued meromorphic function on the whole $(\lambda, \mu, \nu)$-space $\mathbb{C}^{\ell+2 m}$.

This theorem can be shown by an argument similar to that in Bernstein-Gel' fand [BG], where Hironaka's desingularization theorem plays a central role. This also follows from the 
existence of b-functions of multi-parameters which is stated as follows.

Theorem 2 (Kashiwara-Kawai [KK], Sabbah [S]) Let $h_{i}(x)$ (1 $\leq i \leq \ell)$ be polynomials of $x=\left(x_{1} \ldots x_{n}\right)$ and define $a$ multi-valued analytic function $\phi_{\lambda}(x)=\prod_{i=1}^{\ell} h_{i}(x)^{\lambda}$ on an open subset $x=\left\{x \in \mathbb{C}^{n} ; h_{i}(x) \neq 0(1 \leq i \leq \ell)\right\}$ of $\mathbb{C}^{n}$. Then for each $i(1 \leq i \leq \ell)$, there exist a polynomial $P_{i}(\lambda)$ of $\lambda$ whose coefficients are differential operators of the variable $x$ and a non-zero polynomial $b_{i}(\lambda) \in \mathbb{C}[\lambda]$ such that $P_{i}(\lambda)\left(h_{i}(x) \phi_{\lambda}(x)\right)=b_{i}(\lambda) \phi_{\lambda}(x)$ holds on $x$.

Remark 3. Theorem 2 is a generalization to multi-parameter case of Bernstein's result on the existence of b-functions. Theorem 2 as well as its references is communicated to the author by M. Kashiwara.

Under the situation of Theorem 1, it seems difficult to decide whether the condition (A) holds for the given polvnomials $f_{1}(x), \ldots, f_{\ell}(x), g_{1}(x), \ldots, g_{m}(x)$ or nat. In the rest of this section, we give some examples which agree with the condition (A).

Example 1. $x_{+}^{\lambda}(x+\sqrt{-1} y)^{\mu}$

First note that $(x+\sqrt{-1} y)^{\mu}$ defines a continuous function on $\left\{(x, y) \in \mathbb{R}^{2} ; x \geq 0\right\}$ if $\operatorname{Re} \mu>0$. So Theorem 1 can is applicable and we conclude that $x_{+}^{\lambda}(x+\sqrt{-1} y)^{\mu}$ defines 
distributions on $\mathbb{R}^{2}$ meromorphically depending on the parameters $\lambda, \mu$.

Example 2: Let $P(x), Q(x)$ be polynomials of $x=\left(x_{1}, \ldots, x_{n}\right)$. Assume that $P(x) \geq 0$ for all $x$. Let. $\phi_{\lambda, \mu}(x)$ be a branch of $(P(x)+\sqrt{-1} Q(x))^{\lambda}(P(x)-\sqrt{-1} Q(x))^{\mu}$. Then it is easy to see that $\phi_{\lambda, \mu}(x)$ is a single-valued continuous function if $\operatorname{Re}(\lambda+\mu)>0$. So, by Theorem 1 . $\phi_{\lambda, \mu}(x)$ is extended to a $0^{-}\left(\mathbb{R}^{n}\right)$-valued distribution on the whole $(\lambda, \mu)$-space.

Example 3. Put $f(x, y, t)=1+2 z^{2}+\left(\sqrt{-1} t-|z|^{2}\right)^{2}$ $(z=x+\sqrt{-1} y))$. If $\operatorname{Re}(\lambda+\mu)>0$, each branch of the function $f(x, y, t)^{\lambda} \overline{f(x, y, t)}^{\mu}$ defines a single-valued continuous function on $\mathbb{R}^{3}$. This is shown in the following way. First note that

$$
f(x, y, t)=\frac{1}{2}\left(1+|z|^{2}-\sqrt{-1} t\right)^{2}(1+h(x, y, t)),
$$

where

$$
h(x, y, t)=\frac{\left(1-|z|^{2}+\sqrt{-1} t\right)^{2}+4 z^{2}}{\left(1+|z|^{2}-\sqrt{-1} t\right)^{2}} .
$$

A simple calculation shows the inequality $|h(x, y, t)| \leq 1$. Therefore $\operatorname{Re}(1+h(x, y, t)) \geq 0$ for all $(x, y, t) \in \mathbb{R}^{3}$. So each branch of $(1+h(x, y, t))^{1 / 2}$ is single-valued and

$$
f(x, y, t)^{1 / 2}=\frac{1}{\sqrt{2}}\left(1+|z|^{2}-\sqrt{-1} t\right)(1+h(x, y, t))^{1 / 2} .
$$


Then the claim follows. Applying Theorem 1, we find that $f(x, y, t)^{\lambda}{\overline{f(x, y, t)^{\mu}}}^{\mu}$ defines a family of distributions on $\mathbb{E}^{3}$ meromorphically depending on $(\lambda, \mu)$. This example is a special case of Theorem 9 in $\$ 2$. Moreover, it is an interesting problem to regularize the divergent integral $\int f(x, y, z)^{\lambda} \overline{f(x, y, z)}^{\mu} d x d y d z$ (cf. Remark 13 (iii)).

\section{\$2. An application of Theorem 1.}

Take positive integers $p, q$ and put $n=2 p+q$. Define

$$
G=\left\{g \in S L(n, \mathbb{C}) ; \quad t_{\bar{g}} S_{p q} g=S_{p q}\right\} \text {, }
$$

where $S_{p q}=\left[\begin{array}{lll} & & J_{p} \\ & I_{q} & \\ J_{p} & & \end{array}\right]$ with $J_{p}=\left[\begin{array}{cc}\cdot & 1 \\ v & \end{array}\right]$ a $p \times p$

matrix. By definition, $G \simeq S U(p+q, p)$. Take mutually commuting involutions $\theta, \sigma$ of $G$ defined by $\theta(g)={ }^{t} \bar{g}^{-1}, \sigma(g)=\bar{g}$ for any $g \in G$. Using $\theta, \sigma$, define $K=\{g \in G ; \theta(g)=g\}$ and $H=\{g \in G ; \sigma(g)=g\}$. Then, clearly we have $K \simeq S(U(p+q) \times U(p)), \quad H \simeq S O(p+q, p)$ and in particular, $K$ is a maximal compact subgroup of $G$. Let $g$ be the Lie algebra of G.

Introduce some notation:

$$
\begin{aligned}
& \underline{a}_{p}=\{x \in \underline{g} ; x \text { is a real diagonal matrix }\} \\
&=\left\{\operatorname{diag}\left(t_{1}, \ldots, t_{p}, 0, \ldots, 0,-t_{p}, \ldots, t_{1}\right) ;\right. \\
&\left.t_{1}, \ldots, t_{p} \in \mathbb{R}\right\}
\end{aligned}
$$




$$
\begin{aligned}
& \underline{n}=\{x \in \underline{g} ; \quad x \text { is upper triangular and nilpotent }\} \\
& \underline{n}=\{x \in \underline{g} ; x \text { is lower triangular and nilpotent }\} \\
& \underline{k}=\text { Lie algebra of } K \\
& \underline{h}=\text { Lie algebra of } H \\
& \underline{m}=Z_{\underline{k}}\left(\underline{a}_{\underline{p}}\right) \\
& \underline{M}=Z_{K}\left(\underline{a}_{\underline{p}}\right) \\
& A_{\underline{p}}=\exp \underline{a}_{\underline{p}}, \quad N=\exp \underline{n}, \bar{N}=\exp \underline{\underline{n}} \\
& H_{O} \text { : The identity component of } H .
\end{aligned}
$$

For a matrix $g=\left(g_{i j}\right)_{1 \leq i, j \leq n}$, let $D_{k}(g)$ denote the determinant of the matrix $\left(g_{i j}\right)_{1 \leq i, j \leq k}$. Using these, define functions $f_{k}(g)=D_{k}\left(\sigma(g)^{-1} g\right)$ for any $g \in G$. The next lemma follows from the definition.

Lemma 4. (i) $f_{k}(h g)=f_{k}(g)$ for any $h \in H \quad(k=.1, \ldots$, $n-1)$

(ii) $\overline{f_{, k}(g)}=f_{n-k}(g) \quad(k=1, \ldots, p)$.

Let $\tilde{G}$ be the universal covering group of $G$ and let $\pi: \widetilde{G} \rightarrow G$ be a natural projection. Let $\tilde{K}, \tilde{H}$ be the analytic subgroups of $\widetilde{G}$ corresponding to $k$, $h$, respectively. Put $\widetilde{A}_{P}$ $=\exp a_{\underline{p}}, \tilde{N}=\exp \underline{n}, \bar{N}^{2}=\exp \underline{n}$ in $\tilde{G}$ and $\tilde{M}=z_{\widetilde{K}}\left(\underline{a}_{\underline{p}}\right)$.

A simple calculation shows that

$$
\begin{gathered}
\tilde{M} \simeq\left\{\left(\phi, \alpha_{1}, \ldots, \alpha_{p}, m\right) ; \phi \in \mathbb{R}, \alpha_{i} \in \mathbb{C},\left|\alpha_{i}\right|=1(1 \leq i \leq p),\right. \\
m: \text { a unitary matrix of degree } \\
\alpha_{1} \cdots \alpha_{p}=e^{-n \sqrt{-1} \phi}=\operatorname{det} m
\end{gathered}
$$$$
m=\left\{\left(\phi, \theta_{1}, \ldots, \theta_{p}, x\right) ; \phi, \theta_{1}, \ldots, \theta_{p} \in \mathbb{R}\right.
$$ 


$$
\begin{aligned}
& x \text { : a skew Hermitian matrix of degree } \\
& \sqrt{-1}\left(\theta_{1}+\ldots+\theta_{p}\right)=-\sqrt{-1} r^{\prime}=-\operatorname{tr} x_{i}
\end{aligned}
$$

and that under this identification

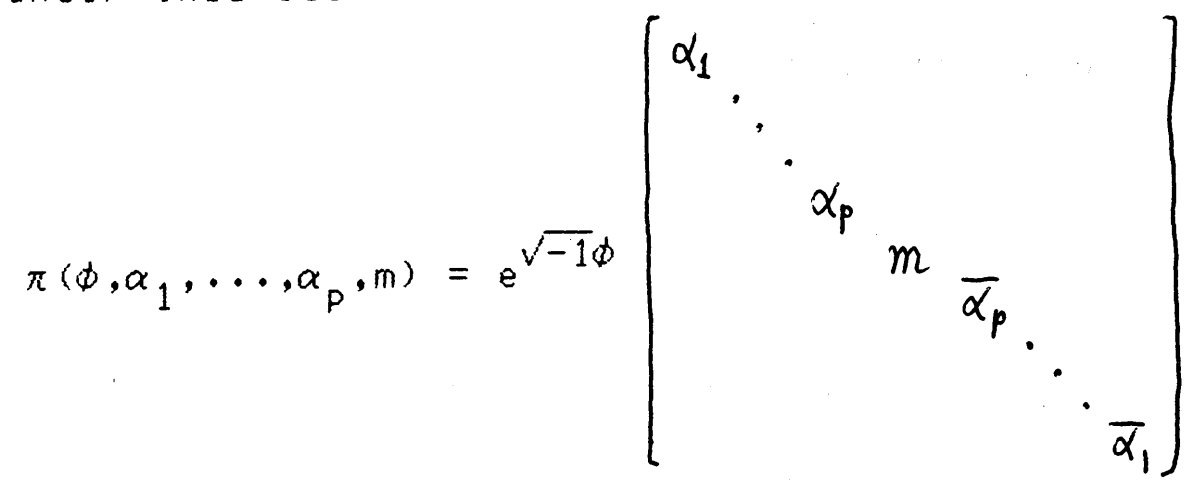

for any $\left(\phi, \alpha_{1}, \ldots, \alpha_{p}, m\right) \in \tilde{M}$. Since $\tilde{M}$ is reductive, there are many one-dimensional representations of $\tilde{M}$. In particular. define representations $x_{j}(1 \leq j \leq p)$ of $\tilde{M}$ by

$$
\chi_{j}\left(\phi, \alpha_{1}, \ldots, \alpha_{p}, m\right)=e^{2 j \sqrt{-1} \phi}\left(\alpha_{1} \cdots \alpha_{j}\right)^{2}
$$

for any $\left(\phi, \alpha_{1}, \ldots, \alpha_{p}, m\right) \in \tilde{M}$. Their differentials are denoted by $\delta x_{j}(1 \leq j \leq p)$. Then

$$
\delta \chi_{j}\left(\phi, \theta_{1}, \ldots, \theta_{p}, x\right)=\sqrt{-1}\left(\theta_{1}+\cdots+\theta_{j}+2 j \phi\right)
$$

for any $\left(\phi, \theta_{1}, \ldots, \theta_{p}, x\right) \in \underline{m}$.

Lemma 5. Assume that $1 \leq j \leq p-1$. Then $\mu \delta x_{j}$ is 1 ifted to a character of $\tilde{M}$ if and only if $\mu \in \mathbb{Z}$. On the other hand, $x_{P}(\exp Y)^{\mu}=\exp \mu \delta x_{P}(Y)(Y \in m)$ defines a character of $\tilde{M}$ 
for any $u \in \mathbb{C}$.

This lemma follows from the concrete form of $\tilde{M}$ given before.

For simplicity, put

$$
a(t)=\exp \left(\operatorname{diag}\left(t_{1}, \ldots, t_{p}, 0, \ldots, 0,-t_{p}, \ldots,-t_{1}\right)\right) \in \tilde{A}_{p}
$$

for any $t_{1}, \ldots, t_{p} \in \mathbb{R}$ and $\tilde{f}_{j}=f_{j}{ }^{\circ} \pi(1 \leq j \leq n-1)$. The next lemma follows from the definition.

Lemma 6. If $1 \leq j \leq P$, then

$$
\tilde{f}_{j}(g m a(t) n)=e^{2 t} 1^{+\cdots+2 t} j_{\chi_{j}(m)} \tilde{f}_{j}(g)
$$

for any $g \in \tilde{G}, m \in \tilde{M}, \quad a(t) \in \tilde{A}_{P}, \quad n \in \tilde{N}$.

Lemma 7. (i) The set $\Omega=\tilde{H} \tilde{M} \tilde{A}_{p} \tilde{N}$ is connected and open dense in $\widetilde{G}$.

$$
\text { (ii) } \Omega=\left\{g \in \widetilde{G} ; \tilde{f}_{j}(g) \neq 0(1 \leq j \leq p)\right\} \text {. }
$$

Proof. Since $\tilde{H}, \tilde{M}, \tilde{A}_{P}, \tilde{N}$ are all connected, so is $\Omega$. That $\Omega$ is open dense in $\tilde{G}$ is shown by direct calculation or follows from [M]. (ii) is a special case of [0S2].

The next proposition is the key to the subsequent theorem.

Proposition 8. Take $\lambda \in \mathbb{C}$ with $\operatorname{Re} \lambda>0$. 
(i) Suppose that $1 \leq j<p$. Then $\tilde{f}_{j}(g)^{\lambda}$ is single-valued if and only if $\lambda$ is an integer.

(ii) Each branch of $\tilde{f}_{P}(g)^{\lambda}$ defines a single-valued continuous function on $\widetilde{G}$.

Proof. (i) The "only if" part is clear. To prove the "If" part, consider the case $j=1$. For any $z \in \mathbb{C}, \bar{n}_{1}(z)=$

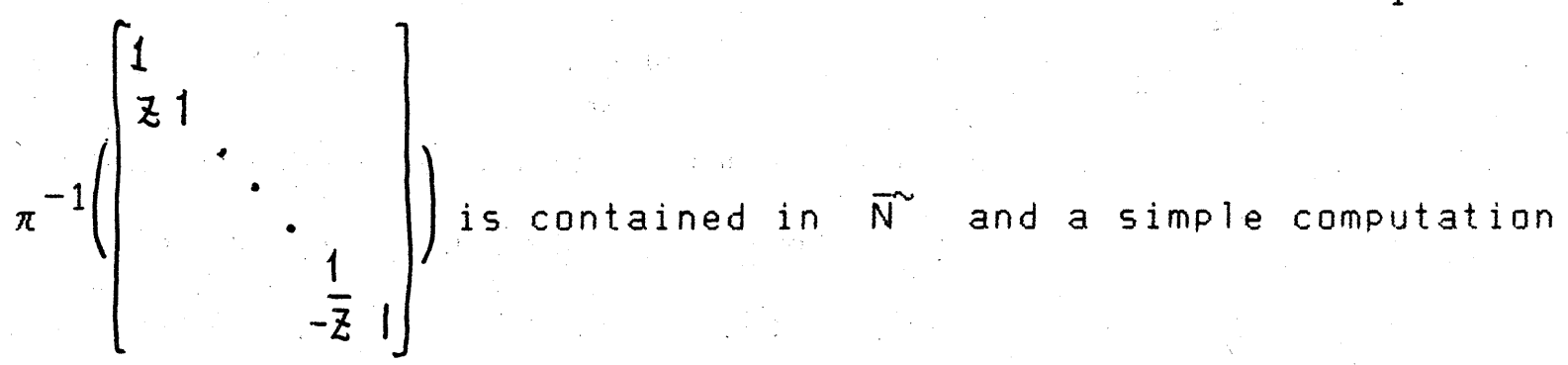
shows that $\tilde{f}_{p}\left(\bar{n}_{1}(z)\right)=1+z^{2}$. If $\lambda$ is not an integer, the function $\left(1+z^{2}\right)^{\lambda}$ is not single-valued and therefore $\tilde{f}_{p}(g)^{\lambda}$ is not single-valued. So the resule follows when $j=1$. The other cases are shown by a similar way.

(ii) Define a function $\Phi_{\lambda}(g)$ on $\widetilde{G}$ as follows. If $g=h m a(t) n$ with $h \in \tilde{H}, m=\exp (Y), Y \in m, a(t) \in \tilde{A}_{p}$, $n \in \tilde{N}$, then $\Phi_{\lambda}(g)=\exp \left\{\lambda \delta \chi_{p}(Y)+2 \lambda\left(t_{1}+\cdots+t_{p}\right)\right\}$ and if $g \notin \Omega$, then $\Phi_{\lambda}(g)=0$. Since $\tilde{M} \simeq M_{1} \times \mathbb{R}$ for a connected compact Lie group $M_{1}, \Phi_{\lambda}(g)$ is well-defined. First show that $\Phi_{\lambda}(g)$ is continuous on $\widetilde{G}-s$, where $s=\left\{g \in \widetilde{G} ; \prod_{j=1}^{p-1} \tilde{f}_{j}(g)=\right.$ 03. Since $\Omega$ is connected, it easily follows from the definition that $\Phi_{\lambda}(g)$ is continuous on $\Omega$. On the other hand, since $\left|\Phi_{\lambda}(g)\right|=\left|\tilde{f}_{p}(g)\right|^{\operatorname{Re} \lambda}$ if $g \in \Omega$ and since $\tilde{G}-$ (SUS) (C $\left.\left\{\tilde{f}_{p}(g)=0\right\}\right)$ is the boundary of $\Omega$ in $\tilde{G}-s$, we find that $\Phi_{\lambda}(g)$ is continuous on $\tilde{G}-S$. Next show that $\Phi_{\lambda}(g)$ is actually continuous on $\tilde{G}$. Now take $j(1 \leq j<p)$ and $f i x$ it. 
By the concrete form of $\tilde{f}_{j}(\bar{n})$, we find that $\operatorname{codim} \bar{N}^{\sim}\left\{\bar{n} \in \bar{N}^{2}\right.$; $\left.\widetilde{f}_{j}(\bar{n})=0\right\} \geq 2$. Put $\tilde{P}=\tilde{M} \tilde{A}_{P} \tilde{N}$ for simplicity. Then for any $\bar{n} \in \bar{N}, p \in \tilde{P}, \tilde{f}_{j}(\bar{n} p)=0$ if and only if $\tilde{f}_{j}(\bar{n})=0$. So we find that $\operatorname{codim} \bar{N}_{\bar{P}} S_{j} \cap \bar{N} \widetilde{P} \geq 2$, where $S_{j}=$ $\left\{g \in \widetilde{G} ; \widetilde{f}_{j}(g)=0\right\}$. On the other hand, the complement of $\bar{N} \widetilde{P}$ in $\widetilde{G}$ has also codimension $\geq 2$ in $\widetilde{G}$. So $\operatorname{codim}_{\widetilde{G}} S_{j} \geq 2$.

Since $S=\bigcup_{j=1}^{P-1} S_{j}$, we find that $\operatorname{codim}_{\widetilde{G}} S \geq 2$. Now take $g \in S$ with $\tilde{f}_{p}(g) \neq 0$. Let $U$ be a simply connected neighbourhood of $g$ such that $\tilde{f}_{p}$ is invertible on it. Noting that $\Phi_{\lambda}(g)$ is locally a branch of the multi-valued function $\tilde{f}_{p}(g)^{\lambda}$, take a single-valued branch $\Phi_{\lambda}(g)$ of $\widetilde{f}_{p}(g)^{\lambda}$ on $U$ coinciding with $\Phi_{\lambda}(g)$ on $U-S$. Since $\operatorname{codim}_{\tilde{G}} S \geq 2, U-S$ is connected. Hence $\Phi_{\lambda}(g)$ and $\Phi_{\lambda}^{\prime}(g)$ must coincide on $U$. Then $\Phi_{\lambda}(g)$ defines a single-valued continuous function on $\widetilde{G}$. By definition, $\Phi_{\lambda}(g)$ is the branch of $\tilde{f}_{p}(g)^{\lambda}$ with $\Phi_{\lambda}(e)=1$. Therefore, each branch of $\tilde{f}_{p}(g)^{\lambda}$ is a constant multiple of $\Phi_{\lambda}(g)$ and the result follows. QED

For $\alpha, \beta \in \mathbb{C}$ with $\operatorname{Re}(\alpha+\beta)>0$, let $\eta_{\alpha, \beta}(g)$ be the branch of $\tilde{f}_{p}(g) \alpha_{\tilde{f}_{p}(g)}^{\beta}$ such that $\eta_{\alpha, \beta}(e)=1$. Then it follows from proposition 8 that $\eta_{\alpha, \beta}(g)$ is a single-valued continuous function on $\widetilde{G}$. Take $\ell_{1}, \ldots, \ell_{p-1} \in \mathbb{Z}, m_{1}, \ldots$ $m_{q} \in \mathbb{N}$ and $f i x$ them. For any $\lambda=\left(\lambda_{1}, \ldots, \lambda_{p-1}\right) \in \mathbb{C}^{p-1}, \alpha$, $\beta \in \mathbb{C}$, define a function $\Phi_{\lambda, \alpha, \beta}(g)$ on $\widetilde{G}$ with parameters $(\lambda, \alpha, \beta) \in \mathbb{C}^{p-1} \times \mathbb{C}^{2}$ as follows. If $g \in \Omega$, then 


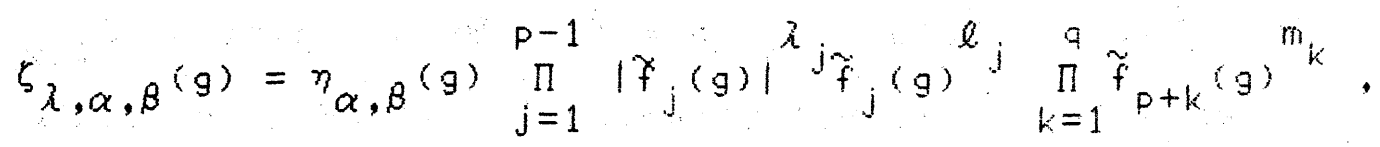

and $\zeta_{\lambda, \alpha, \beta}(g)=0$ if otherwise.

Theorem 9. Fix $\ell_{1}, \ldots, \ell_{p-1} \in \mathbb{Z}$ and $m_{1}, \ldots, m_{q} \in \mathbb{N}$.

Then the function $\zeta_{\lambda, \alpha, \beta}(g)$ on $\widetilde{G}$ with parameters $(\lambda, \alpha, \beta) \in \mathbb{C}^{p-1} \times \mathbb{C}^{2}$ satisfies the following properties.

(i) If $\operatorname{Re}\left(\lambda_{i}+\ell_{i}\right)>0(1 \leq i<p)$ and $\operatorname{Re}(\alpha+\beta)>0$, then $\zeta_{\lambda, \alpha, \beta}(g)$ is a single-valued continuous function on $\tilde{G}$.

(ii) As a function of $(\lambda, \alpha, \beta), \zeta_{\lambda, \alpha, \beta}$ (g) is extended to a $0^{-}(\widetilde{G})$-valued meromorphic function on the whole $(\lambda, \alpha, \beta)-p$ lane.

Proof. The idea of the proof is same as in [0S2] extending that in $\cos 1]$.

(i) follows from Proposition 8 .

(ii) Since $\prod_{i=1}^{q} f_{i}(g)^{m_{i}}$ is real analytic and since the product of a distribution and a real analytic function is a distribution, to prove the theorem, it suffices to show the case where $m_{1}=\ldots=m_{q}=0$. So assume the condition $m_{1}=\ldots=$ $m_{q}=0$. As in the proof of Proposition 8, put $\tilde{P}=\tilde{M} \tilde{A}_{p} \tilde{N}$. Since $g \bar{N}^{\sim} \tilde{P}$ is an open subset of $\tilde{G}$ for any $g \in \tilde{G}$, it also suffices to show that the restriction of $\zeta_{\lambda, \alpha, \beta}$ to $g \bar{N}^{\sim} \widetilde{P}$ can be extended to a $D^{-}\left(g \bar{N}^{\sim} \widetilde{P}\right)$-valued meromorphic function of $(\lambda, \alpha, \beta)$. Let $\bar{N}^{2} \times \widetilde{P} \longrightarrow g \bar{N}^{\sim} \widetilde{P}$ be a natural product map and consider the pull back $\zeta_{\lambda, \alpha, \beta}^{\star}$ of $\zeta_{\lambda, \alpha, \beta}$ for this map. Then it follows

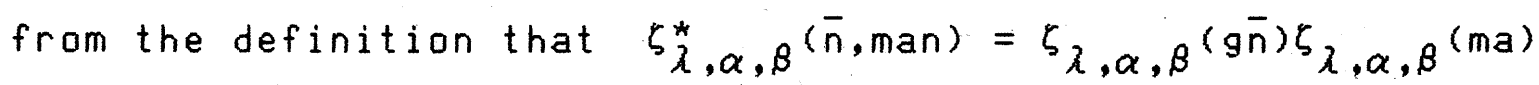


$\left(\bar{n} \in \bar{N}^{2}, m \in \tilde{M}, a \in \tilde{A}_{P}, n \in \tilde{N}\right)$. By definition, $\zeta_{\lambda, \alpha, \beta^{(m a)} \text { is }}$ invertible and is holomorphically extended to $(\lambda, \alpha, \beta)-p l a n e$. On the other hand, take a base $x_{1}, \ldots x_{d}$ of $\bar{n}(d=\operatorname{dim} \bar{n})$ and put $\zeta_{\lambda, \alpha, \beta}\left(x_{1}, \ldots, x_{d}\right)=\zeta_{\lambda, \alpha, \beta}\left(g^{\bar{n}}(x)\right)$, where $\bar{n}(x)=\exp \left(x_{1} x_{1}+\ldots+x_{d} x_{d}\right)$. Since $\bar{f}_{j}(g \bar{n}(x))(1 \leq j \leq p)$ are complex-valued polynomials, Theorem 1 combined with Proposition 8 implies that $\zeta_{\lambda, \alpha, \beta}\left(g^{\bar{n}}\right)$ can be extended to a meromorphic function of $(\lambda, \alpha, \beta)$. Hence the theorem follows. QED

\section{\$3. Properties of functions related with the distribution $\eta_{\alpha, \beta}$.}

In this section, we restrict our attention to the case $P=$ 1. namely, the case $\widetilde{G}=$ the universal covering group of

SU $(q+1,1)$. We have already defined the distribution $\eta_{\alpha, \beta}(g)$ in the previous section. Put

$$
\phi_{\alpha, \beta}(g)=\int_{K_{s}} \eta_{\alpha, \beta}(g k) d k \text {, }
$$

where $k_{s}=(\widetilde{K}, \widetilde{K})$ and $d k$ is the Haar measure on it normalized by $\int_{K_{S}} d k=1$. The purpose of this section is to study this function in detail. As a consequence, we shall compute the value of "a regularization in a certain sense of the divergent integral

$$
I(\alpha, \beta)=\int_{\bar{N}} \eta_{\alpha, \beta}(\bar{n}) d \bar{n} \cdot "
$$

(Cf. Theorem 12.) T. Oshima [0] introduced c-functions for 
semisimple symmetric spaces, generalizing Harish-Chandra's c-function for Riemannian symmetric spaces. In the case of the univeral covering space of $S U(p+1, q+1) / S O(p+1, q+1)$, the c-function is a regularization of the divergent integral $\int_{\bar{N}^{2}} \zeta_{\lambda, \alpha, \beta}(\bar{n}) d \bar{n}$ in some sense and the above integral $I(\alpha, \beta)$ is its special case. (See Remark 14 in this section.)

Let $E_{i j}$ be a matrix of degree $q+2$ whose $(i, j$, ) entry is 1 if $(i, j)=,(i, j)$ and 0 if otherwise. Then $\underline{g}_{c}=$ the complexification of $g$ ) is spanned by $E_{i j}$ ( $\left.i \neq j\right), E_{i j}-E_{j j}$. Let $U(\underline{g})$ be the universal enveloping algebra of $\underline{g}_{c}$ and let $\omega$ be its Casimir element. Then, by definition, we have

$$
2(q+2) \omega=\sum_{i<j}\left\{\left(E_{i i}-E_{j j}\right)^{2}+2(q+2) E_{j i} E_{i j}\right\}+(q+2) \sum(q+1-2 j) E_{j j} \cdot
$$

In the sequel, we identify elements of $\underline{g}_{c}$ with left invariant vector fields on $\tilde{G}$ and also identify elements of U(g) with left invariant differential operators on $\widetilde{G}$. By direct calculation, we have

Lemma 10. $\quad \omega \eta_{\alpha, \beta}=\left\{(\alpha+\beta)(\alpha+\beta+q+1)+\frac{q}{q+2}(\alpha-\beta)^{2}\right\} \eta_{\alpha, \beta}$.

We now start to study the function $\phi_{\alpha, \beta}$. In the sequel, $i=\sqrt{-1}$ unless otherwise stated.

Proposition 11. (i) $\phi_{\alpha, \beta}$ (g) is ( $\left.\tilde{H}, K_{5}\right)$-invariant. (ii) $\quad \omega \phi_{\alpha, \beta}=\left\{(\alpha+\beta)(\alpha+\beta+q+1)+\frac{q}{q+2}(\alpha-\beta)^{2}\right\} \phi_{\alpha, \beta}$. (iii) $\phi_{\alpha, \beta}$ is real analytic. 
(iv) Put $S_{\alpha, \beta}(t)=\phi_{\alpha, \beta}(\exp t Y)$, where $Y=$ $\frac{1}{2}\left(E_{11}-E_{q+2, q+2}\right)$. Then $S_{\alpha, \beta}(-t)=S_{\alpha, \beta}(t)$ and if $\operatorname{Re}(\alpha+\beta)>0$, the following integral formula holds:

$$
\begin{aligned}
s_{\alpha, \beta}(t)=\frac{1}{a_{q}} & \int_{\mathbb{R} \times \mathbb{C}^{q}} \frac{\left\{e^{t}+2(z, z)+e^{-t}\left(|z|^{2}-i x\right)^{2}\right\}^{\alpha}}{\left(1+|z|^{2}-i x\right)^{2 \alpha+q+1}} \\
& \times \frac{\left\{e^{t}+2 \overline{(z, z)}+e^{-t}\left(|z|^{2}+i x\right)^{2}\right\}^{\beta}}{\left(1+|z|^{2}+i x\right)^{2 \beta+q+1}} d x d z d \bar{z},
\end{aligned}
$$

where, for any $z=\left(z_{1}, \ldots, z_{q}\right) \in \mathbb{C}^{q},(z, z)=z_{1}{ }^{2}+\ldots+z_{q}{ }^{2}$ and $|z|^{2}=\left|z_{1}\right|^{2}+\ldots+\left|z_{q}\right|^{2}$ and $d z d \bar{z}=d z_{1} d \bar{z}_{1} \cdots d z_{q} d \bar{z}_{q}$. Moreover,

$$
a_{q}=\int_{\mathbb{R} \times \mathbb{C}^{q}}\left\{\left(1+|z|^{2}\right)^{2}+x^{2}\right\}^{-q-1} d x d z d \bar{z}=\frac{(-\pi i)^{q} \pi}{2^{q} \Gamma(q+1)} \text {. }
$$

Proof. (i) is clear from the definition. Since $w$ is contained in the center of $U(g)$ and since $\phi_{\alpha, \beta}$ is obtained by integrating $\eta_{\alpha, \beta}$ over $K_{s}$, (ii) follows from Lemma 10 . Noting that $\phi_{\alpha, \beta}$ is relative invariant by the right $\tilde{k}$-action, we find that (ii) implies (iii). Using a standard technique of changing the integration over $K_{s}$ by that over $\tilde{N}^{2}$, we obtain the integral formula in (iv). It is easy to check that the integral in the right side is actually convergent if $\operatorname{Re}(\alpha+\beta)>$ 0. QED

Theorem 12 .

(i) $S_{\alpha, \beta}(t)=2^{-\alpha-\beta} \frac{\Gamma\left(\frac{q+2}{2}\right) \Gamma\left(\alpha+\beta+\frac{q+2}{2}\right)}{\Gamma\left(\alpha+\frac{q+2}{2}\right) \Gamma\left(\beta+\frac{q+2}{2}\right)}(\text { ch t })^{\alpha+\beta}$

$\times F\left(-\alpha,-\beta, \frac{q+1}{2} ;(\text { th } t)^{2}\right)$. 
(ii) If $\operatorname{Re}(\alpha+\beta)>-\frac{q+1}{2}$, then

(4)

$$
\lim _{t \rightarrow+\infty} e^{-(\alpha+\beta) t} S_{\alpha, \beta}(t)=2^{-2 \alpha-2 \beta} \frac{\Gamma(q+1) \Gamma(2 \alpha+2 \beta+q+1)}{\Gamma(2 \alpha+q+1) \Gamma(2 \beta+q+1)}
$$

On the other hand, if $\operatorname{Re}(\alpha+\beta)<-\frac{q+1}{2}$, then

$$
\begin{gathered}
\lim _{t \rightarrow+\infty} e^{(\alpha+\beta+q+1) t} S_{\alpha, \beta}(t) \\
\quad=\frac{2}{\sqrt{\pi}} \frac{\sin \pi \alpha \sin \pi \beta}{\sin \pi\left(\alpha+\beta+\frac{q-1}{2}\right)} \frac{\Gamma(q+1) \Gamma(\alpha+1) \Gamma(\beta+1) \Gamma\left(\alpha+\beta+\frac{q+2}{2}\right)}{\Gamma\left(\alpha+\frac{q+2}{2}\right) \Gamma\left(\beta+\frac{q+2}{2}\right) \Gamma\left(\alpha+\beta+\frac{q+3}{2}\right)} .
\end{gathered}
$$

Proof: First of all, put $r=\frac{q+1}{2}$ for simplicity. Modifying the technique of calculating the radial component of the Casimir operator ( $c f .[W, p .277]$ ), we can obtain the concrete form of $\left(\omega \phi_{\alpha, \beta}\right)$ (exp ty). Then Proposition 11, (ii) leads to

$$
\left\{\frac{d^{2}}{d t^{2}}+\left(q \frac{c h t}{s h t}+\frac{s h t}{c h t}\right) \frac{d}{d t}+\frac{(\alpha-\beta)^{2}}{(c h t)^{2}}-(\alpha+\beta)(\alpha+\beta+q+1)\right\} s_{\alpha, \beta}(t)=0 .
$$

Put $x=(c h t)^{2}$ and $T_{\alpha, \beta}(x)=S_{\alpha, \beta}(t)$. Then $T_{\alpha, \beta}(x)$ satisfies the differential equation

$$
\left\{x(x-1) \frac{d^{2}}{d x^{2}}+((r+1) x-1) \frac{d^{2}}{d x}+\frac{(\alpha-\beta)^{2}}{4 x}-\frac{1}{4}(\alpha+\beta)(\alpha+\beta+2 r)\right\} u(x)=0 .
$$

Each solution of this differential equation which is real analytic in a neighbourhood of $t=0$, that is, in a neighbourhood of $x=1$ equals to $x^{(\alpha+\beta) / 2} F\left(-\alpha,-\beta, r: \frac{x-1}{x}\right)$ up to a constant factor. Therefore we find that 


$$
s_{\alpha, \beta}(t)=a(\alpha, \beta)(c h t)^{\alpha+\beta} F\left(-\alpha,-\beta, r ;(t h t)^{2}\right)
$$

for some constant $a(\alpha, \beta)$ depending on $\alpha, \beta$ meromorphically. In virtue of the integral representation (3) of $S_{\alpha, \beta}{ }^{(t)}$ and the central limit theorem, we have

$$
\begin{aligned}
& \lim _{t \rightarrow+\infty} e^{-(\alpha+\beta) t_{S}}{ }_{\alpha, \beta}(t) \\
&= \frac{1}{a_{q}} \int_{\mathbb{R} \times \mathbb{C}^{q}}\left(1+|z|^{2}-i x\right)^{-2 \alpha-2 r}\left(1+|z|^{2}+i x\right)^{-2 \beta-2 r} d x d z d \bar{z} \\
&= \frac{1}{a_{q}} \int_{\mathbb{C}^{q}}\left(1+|z|^{2}\right)^{-2 \alpha-2 \beta-2 r+1} d z d \bar{z} \\
& \times \int_{-\infty}^{\infty}(1-i x)^{-2 \alpha-2 r}(1+i x)^{-2 \beta-2 r} d x
\end{aligned}
$$

under the condition $\operatorname{Re}(\alpha+\beta)>0$. A simple computation shows that

$$
\int_{\mathbb{C}^{q}}\left(1+|z|^{2}\right)-2 \alpha-2 \beta-2 r+1 d z d \bar{z}=(-2 \pi i)^{9} \frac{\Gamma(2 \alpha+2 \beta+2 r)}{\Gamma(2 \alpha+2 \beta+4 r-1)}
$$

if $\operatorname{Re}(\alpha+\beta)>0$. On the other hand, it follows from [E, $p .12$. formula $(30)$ ] that

$$
\begin{aligned}
& \int_{-\infty}^{\infty}(1-i x)^{-2 \alpha-2 r(1+i x)^{-2 \beta-2 r} d x} \\
= & 2 \int_{0}^{\pi / 2} \cos 2(\alpha-\beta) \theta(\cos \theta)^{2(\alpha+\beta+2 r-1)} d \theta \quad(x=\tan \theta) \\
= & \frac{2^{-2 \alpha-2 \beta-4 r+4} \pi \Gamma(2 \alpha+2 \beta+4 r-1)}{\Gamma(2 \alpha+2 r) \Gamma(2 \beta+2 r)}
\end{aligned}
$$

if $\operatorname{Re}(\alpha+\beta)>-2 r+\frac{1}{2}$. Therefore we obtain (4) under the 
condition $\operatorname{Re}(\alpha+\beta)>0$. Since both sides of (4) depend on $\alpha, \beta$ meromorphically, (4) holds if $\operatorname{Re}(\alpha+\beta)>-r$.

Now we recall a connection formula for Gaussian hypergeometric functions (cf. [E, p.107. formula (33) ]):

$$
\begin{gathered}
F(a, b, c ; x)=\frac{\Gamma(c) \Gamma(a+b-c)}{\Gamma(a) \Gamma(b)}|1-x|^{c-a-b} F(c-a, c-b, c-a-b+1 ; 1-x) \\
+\frac{\Gamma(c) \Gamma(c-a-b)}{\Gamma(c-a) \Gamma(c-b)} F(a, b, a+b-c+1 ; 1-x)
\end{gathered}
$$

$(0<x<1)$. Substituting $a=-a, b=-\beta, c=r, x=(t h t)^{2}$ in this formula and multiplying both sides by $a(\alpha, \beta)(c h t)^{\alpha+\beta}$, we abtain

(8) $S_{\alpha, \beta}(t)=a(\alpha, \beta) \frac{\Gamma(r) \Gamma(\alpha+\beta+r)}{\Gamma(\alpha+r) \Gamma(\beta+r)}(c h t)^{\alpha+\beta}$

$$
\begin{gathered}
\times F\left(-\alpha,-\beta,-\alpha-\beta-r+1 ;(c h t)^{-2}\right) \\
+a(\alpha, \beta) \frac{\Gamma(r) \Gamma(-\alpha-\beta-r)}{\Gamma(-\alpha) \Gamma(-\beta)}(c h t)^{-\alpha-\beta-2 r} \\
\times
\end{gathered}
$$

If $\operatorname{Re}(\alpha+\beta)>-r$, this formula implies

$$
\lim _{t \rightarrow+\infty} e^{-(\alpha+\beta) t} S_{\alpha, \beta}(t)=2^{-\alpha-\beta} a(\alpha, \beta) \frac{\Gamma(r) \Gamma(\alpha+\beta+r)}{\Gamma(\alpha+r) \Gamma(\beta+r)} .
$$

Comparing this with (4), we have

$$
a(\alpha, \beta)=2^{-\alpha-\beta} \frac{\Gamma\left(r+\frac{1}{2}\right) \Gamma\left(\alpha+\beta+r+\frac{1}{2}\right)}{\Gamma\left(\alpha+r+\frac{1}{2}\right) \Gamma\left(\beta+r+\frac{1}{2}\right)} .
$$

Then (i) follows. Finally, it follows from ( 8 ) that if $\operatorname{Re}(\alpha+\beta)$ $<-r$, then 


$$
\lim _{t \rightarrow+\infty} e^{(\alpha+\beta+2 r) t} S_{\alpha, \beta}(t)=2^{\alpha+\beta+2 r} a(\alpha, \beta) \frac{\Gamma(r) \Gamma(-\alpha-\beta-r)}{\Gamma(-\alpha) \Gamma(-\beta)}
$$

So we obtain (5). QED

Remark 13. (i) The idea of the determination of Theorem 12 (ii) is based on that in [Se1].

(ii) The integral in (7) in the case where $2(\alpha-8) \in \mathbb{Z}$ is treated in H. Schlichtkrull [Sc].

(iii) By changing variables of the integrand in (3), we have

$$
\begin{aligned}
& e^{(\alpha+\beta+q+1) t} a_{q} s_{\alpha, \beta}(t) \\
= & \int_{\mathbb{R} \times \mathbb{C}^{q}} \frac{\left\{1+2(z, z)+\left(|z|^{2}-i x\right)^{2}\right\}^{\alpha}}{\left\{1+e^{-t}\left(|z|^{2}-i x\right)\right\}^{2 \alpha+q+1}} \frac{\left\{1+2 \overline{(z, z)}+\left(|z|^{2}+i x\right)^{2}\right\}^{8}}{\left\{1+e^{-t}\left(|z|^{2}+i x\right)\right\}^{2 \beta+q+1}} d x d z d \bar{z} .
\end{aligned}
$$

If the integration and the limit process were commutative in spite that this is impossible, the divergent integral

(9) $\frac{1}{a_{q}} \int_{\mathbb{R} \times \mathbb{C}^{q}}\left\{1+2(z, z)+\left(|z|^{2}-i x\right)^{2}\right\}^{\alpha}\left(1+2 \overline{(z, z)}+\left(|z|^{2}+i x\right)^{2}\right\}^{\beta} d x d z d \bar{z}$ should coincide with the limit $\lim _{t \rightarrow+\infty} e^{(\alpha+\beta+q+1) t} S_{\alpha, \beta}(t)$ in the case $\operatorname{Re}(\alpha+\beta)<-\frac{q+1}{2}$. For this reason, the right-hand side of (5) is regarded as the value of a regularization of the divergent integral ( 9 ) in a certain sense. It should be noted that if $\operatorname{Re}(\alpha+\beta)>0$, then the integrand of (9) satisfies the condition (A) of Theorem 1. 
Remark 14. Let $G / H$ be a general semisimple symmetric space, where $G$ is a connected semisimple Lie group. T. Oshima [O] introduced c-functions for $G / H$, generalizing Harish-Chandra' $s$ c-function for Riemannian symmetric spaces. In the case where $G$ is linear, he explained four statements i)-iv) in [0,p.365] which in fact give a method of the explicit calculation of the c-function. But to treat the case where $G$ is not linear, we need a modification of the part iv) of $[01$. P.365] as follows.

(B) "If $G / H$ is split rank one, K-relative invariant joint eigenfunctions on $G / H$ of invariant differential operators are expressed by Gaussian hypergeometric functions. (Here $K$ is a closed subgroup of $G$ such that $\mathrm{Ad}_{G}(K)$ is a maximal compact subgroup of $\left.\mathrm{Ad}_{G}(G).\right)$ " We return to our case, namely, $\widetilde{G}=S U(q+1,1)^{2}, \tilde{H}=a$ connected closed subgroup of $\widetilde{G}$ locally isomorphic to $s 0_{0}(q+1,1)$. Then $\left.\psi_{\alpha, \beta}(g)=\phi_{\alpha, \beta^{(g}}{ }^{-1}\right)$ is regarded as a

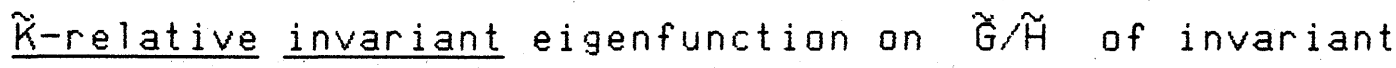
differential operators. Theorem 12 states that $\psi_{\alpha, \beta}(g)$ is actually expressed by Gaussian hypergeametric function and that its asymptotics are determined by using a connection formula of Gaussian hypergeometric functions. In particular, the statement (B) holds for the symmetric space $\tilde{G} / \tilde{H}$.
A proof of the statement
(B) for other cases will be published elsewhere. 


\section{References}

[B] I. N. Bernstein, The analytic continuation of generalized functions with respect to a parameter. Functional Anal. Appl. 6 $(1972), 26-40$.

[BG] I. N. Bernstein, S. I. Gel'fand, The meromorphic property of the function $p^{\lambda}$. Functional Anal. Appl. 3 (1969), 84-86.

[Bj] J-E. Björk, The global homological dimension of some algebras of differential operators, Invent. math., 17 (1972), $67-78$.

[E] A. Erdélyi et al., Higher Transcendental Functions, I. McGraw-Hill Book Company, Inc. 1981.

[K] M. Kashiwara, B-functions and holonomic systems, Invent. math. $38(1976), 33-54$.

[KK] M. Kashiwara, T. Kawai, On holonomic systems for $\prod_{\ell=1}^{N}\left(f_{\ell}+\sqrt{-10}\right)^{\lambda} \ell$, Publ. RIMS, Kyoto Univ. 15 (1979), 551-575. [M] T. Matsuki, The orbits of affine symmetric spaces under the action of minimal parabolic subgroups, J. Math. Soc. Japan 31 (1979), 331-357.

[01] T. Oshima, Fourier analysis on semisimple symmetric spaces, In "Non Commutative Harmonic Analysis and Lie Groups IV" 357-369, Lecture Notes in Math. 880, Springer-Verlag, 1981. [02] T. Oshima, A realization of semisimple symmetric spaces 
and construction of boundary value maps, preprint.

[OS1] T. Oshima, J, Sekiguchi, Eigenspaces of invariant differential aperators on an affine symmetric space, Invent. math. 57 (1980) 1-81.

[0S2] T. Oshima, J. Sekiguchi, In preparation.

[S] C. Sabbah, Proximité évanescente, II. Equations fonctionnelles pour plusieurs fonctions analytiques, to appear.

[Sc] H. Schlichtkrull, One-dimensional K-types in finite dimensional representations of semisimple Lie groups: A generalization of Helgason's theorem, Math. Scand. 54 (1984), $279-294$.

[Se1] J. Sekiguchi, Eigenfunctions of the Laplace-Beltrami operator on a hyperboloid, Nagoya Math. J.79 (1980), 151-185.

[Se2] J. Sekiguchi, Fundamental groups of semisimple symmetric spaces, to appear in Advanced Studies in Pure Math.

[W] G. Warner, Harmonic Analysis on Semi-Simple Lie Groups, II, Springer-Verlag, Berlin-Heidelberg-New York, 1972. 